\title{
Solar Radiation Alters Toxicity of Carbofuran: Evidence from Empirical Trials with Duttaphyrnus melanostictus
}

\author{
M.R.Wijesinghe*, B.A.D.M.C. Jayatillake and W. D. Ratnassoriya \\ Department of Zoology, University of Colombo, Colombo 03.
}

Date Received: 04-04-2011 Date Accepted: 12-09-2011

\begin{abstract}
In the present study we investigated the potential of natural solar radiation to alter the toxicity of a commonly used carbamate pesticide, carbofuran, on tadpoles of the Common Asian Toad Duttaphrynus melanostictus. A single exposure trial was conducted over $96 \mathrm{hrs}$ with three concentrations $(150,250$ and $500 \mathrm{\mu gl}^{-1}$ ) of photo-irradiated and non-irradiated carbofuran. Results show that photo-irradiation markedly reduced the toxicity of carbofuran as evident by its effects on three end points, i.e. mortality, growth and swimming activity. The mortality of tadpoles exposed to irradiated carbofuran was significantly lower than those exposed to the non-irradiated pesticide. Both treatment and control tadpoles showed a hormetic response for mortality. Tadpoles in irradiated tanks were also larger and more active than those in the control tanks. Photo-altered toxicity was evident at all three tested concentrations. The results of this study therefore signals caution when directly linking results of empirical trials to field scenarios and highlight the necessity to evaluate toxic effects of compounds under variable environmental conditions.
\end{abstract}

Keywords: Carbofuran, Duttaphrynus melanostictus, photo-degradation, tadpoles, toxicity

*Correspondence: E-mail-mayuri@zoology.cmb.ac.lk

Tel- + 94071446277

ISSN 2235-9370 Print/ ISSN 2235-9362 Online @2011University of Sri Jayewardenepura 
Wijesinghe et al./Journal of Tropical Forestry and Environment Vol. 01, No. 01 (2011) 48-55

\section{Introduction}

Pesticides are one of the major causes of water pollution and scores of toxicological studies have repeatedly provided evidence implicating that these harmful contaminants are one of the potential causes for the decline of many faunal species. The majority of the standard toxicity tests, while generating a wealth of invaluable information on lethal and sublethal toxic effects observed under controlled conditions, may not directly reflect field scenarios. In recent years increasing attention has been paid to the importance of the non-biological mechanisms that assist in the breakdown of environmental contaminants (e.g. Iesce et al., 2005). One such mechanism is photo-degradation facilitated by UV radiation. With photodegradation occurring in the field, aquatic organisms will most likely be exposed to the photolytic byproducts as opposed to the original compound. Many studies have been carried out on degradation pathways and mechanisms, kinetics, and the characterization of such by-products (Iesce et al., 2005). Accordingly, some have demonstrated that such degradation mechanisms result in the formation of byproducts that may differ from the original substance in terms of environmental persistence and toxicity (Kim et al., 2007, Campanella et al., 2006, Blaustein and Belden, 2003; Oris and Giesy, 1987), although information is lacking for many substances and test organisms.

Sri Lanka is an agricultural country where pesticides are heavily used. These substances are typically washed into water bodies in the form of surface runoff exposing fish and other aquatic organisms to these harmful substances. Nevertheless as Sri Lanka is located close to the equator at $6^{\circ} 55^{\prime} \mathrm{N}$, the country receives intense solar radiation (with a UV index of 11 and above) for at least eight months of the year (WHO 2009). Therefore photo-degradation processes could be expected to assume an important role in the biodegradation of pesticide contaminants. This paper focuses on investigating the potential of natural solar radiation to alter the toxicity of the carbamate pesticide, carbofuran, which is used widely for the control of rice pests (Tennakoon et al., 2009). It was considered particularly important to test the effect of solar radiation on the alteration of toxicity of carbofuran because its peak absorbance (ë max 277$283 \mathrm{~nm}$ ) falls within the UV range of the solar spectrum making it highly photo-degradable (Iesce $e t$ al., 2005). The toxic effects of carbofuran were tested on tadpoles of the bufonid Duttaphrynus melanostictus (Asian Common Toad) (Schneider, 1799).

\section{Methodology}

\subsection{Test organisms and pesticide concentrations}

D. melanostictus tadpoles of Gosner stages 24-25 were collected from three house ponds in the Colombo District in Sri Lanka where no pesticides were used. Collection of larvae from widely separated areas ensured that the larvae collected were from different populations. The larvae were identified as those of $D$. melanostictus by their external morphology and teeth arrangement as described by Kirtisinghe (1995). A commercial preparation of carbofuran (Curater 3G, Hayleys Agro Products Ltd. Colombo, Sri Lanka) was used to prepare low, mid and high test concentrations (150, 250 and $\left.500 \mu \mathrm{gl}^{-1}\right)$. As no published information was available on field levels of carbofuran, test concentrations used in the present study were based on empirical trials conducted elsewhere (e.g. Bretaud et.al., 2002). 


\subsection{Experimental procedure}

A $1000 \mathrm{mgl}^{-1}$ aqueous stock solution of carbofuran was placed in a glass beaker which was irradiated by exposing it to natural solar radiation for five hours between 1000 - $1500 \mathrm{hrs}$ on a day with a clear sky. As Coimbatore, India $\left(11^{\circ} \mathrm{N}\right)$, receives $50 \%$ of the UV radiation between 1000 - $1445 \mathrm{hrs}$ (Balasaraswathy, 2004) the pesticide was irradiated during this period. The UV index at the site of irradiation was measured using a UV index meter (Chaney TM UV index meter, China) every 30 mins. Immediately after, the three pesticide concentrations were prepared using the irradiated stock solution of the pesticide and distilled water.

The larvae were housed in glass tanks ( $n=18$ per tank and $n=56$ per treatment/control) filled with tap water allowed to age for chlorine to dissipate. Appropriate volumes of the pesticide were added to the tanks and the water was mixed using a glass rod. Eighteen tadpoles were then randomly assigned to each tank. A separate set of tanks where tadpoles were exposed to similar concentrations of the non-irradiated pesticide served as the control. Each of the three concentrations of the treatment and control were conducted in triplicate. The experiment was performed as a single exposure trial, which was maintained over the standard $96 \mathrm{hrs}$. A similar experimental procedure has been followed by Zaga et al., (1998) to investigate photo-altered toxicity of carbaryl on amphibian larvae.

The trial was conducted at room temperature $\left(26-28^{\circ} \mathrm{C}\right)$ and inside a dark room. Three fluorescent tubes that do not emit UV radiation (F74 daylight 58W, Tungsten 5", Hungary; zero emissions were confirmed by the UV meter) were used as artificial sources of light. The lights were switched on from $0600-1800 \mathrm{hrs}$ throughout the experimental period to simulate the natural photoperiod (approximately 12 hours light and 12 hours darkness). The larvae were fed once a day with fish food pellets ( $46 \pm 4 \mathrm{mg}$ per day) (Blue Aqua pets, Godagama, Sri Lanka) throughout the trial. All tanks were aerated with a constant flow rate ensuring a continuous supply of oxygen for the larvae. Water temperature, $\mathrm{pH}$ (Water temperature and $\mathrm{pH}$ meter, HM-30v, TOA Electronics Ltd, Tokyo, Japan) and dissolved oxygen (Dissolved Oxygen meter (HANNA HI 9142, Romania) were recorded on Day 2 and Day 4. There were no significant differences in the experimental conditions between the treatment and control tanks as revealed by two sample $\mathrm{t}$-tests performed separately on each parameter i.e. temperature $(\mathrm{t} 33=-1.07, \mathrm{p}>0.05), \mathrm{pH}(\mathrm{t} 26=$ $-1.92, \mathrm{p}>0.05)$ and dissolved oxygen levels $\left(\mathrm{t}_{33}=-1.15, \mathrm{p}>0.05\right)$. Mortality in each tank was monitored daily and body length measurements, staging (according to Gosner, 1960) and recording of swimming activity were conducted on Day 1 and Day 4 . Swimming activity was assessed using a protocol described by Sumanadasa et al. (2008). Qualitative assessments were made of the abnormalities observed in the tadpoles.

\section{Results}

This study reveals that irradiation of carbofuran by natural solar radiation (at an intensity of UV index 10), markedly alters its toxicity and therefore causes less damage to the exposed tadpoles. Mortality of larvae exposed to non-irradiated carbofuran was significantly higher than that observed in those exposed to irradiated carbofuran over the experimental period of four days (Two-way Anova: UV factor - $\mathrm{F}=78.44, \mathrm{p}<0.0001$ : Concentration $-\mathrm{F}=79.62$, $\mathrm{p}<0.0001$ : Interaction $\mathrm{F}=11.21, \mathrm{p}<0.002)$. This trend 
was apparent for all three tested doses of carbofuran with mortality in tanks with irradiated carbofuran being reduced by $15 \%, 29 \%$ and $7 \%$ in 150, 250 and $500 \mu \mathrm{gl}-1$, respectively (Figure 1). In both photoirradiated and non-irradiated tanks the highest mortality levels were recorded from $250 \mu \mathrm{gl}-1$ carbofuran, with levels observed at 150 and $500 \mu \mathrm{gl}-1$ being lower, giving a bell shaped dose-response. Closer examination of mortality patterns revealed that mortality levels also varied with time (Figure 2). In both treatments and the controls there was a gradual increase in the rate of mortality (number of tadpoles dead per day) until the Day 3 after which mortality rates declined.

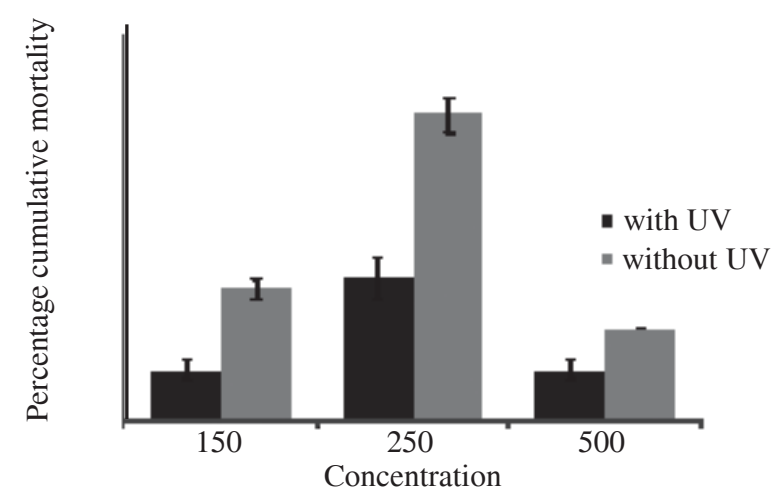

Figure 1: Mortality (Mean \pm S.E.) of D. melanostictus larvae exposed to three concentrations of sunlight irradiated and non-irradiated carbofuran at the end of a four-day single exposure experiment.

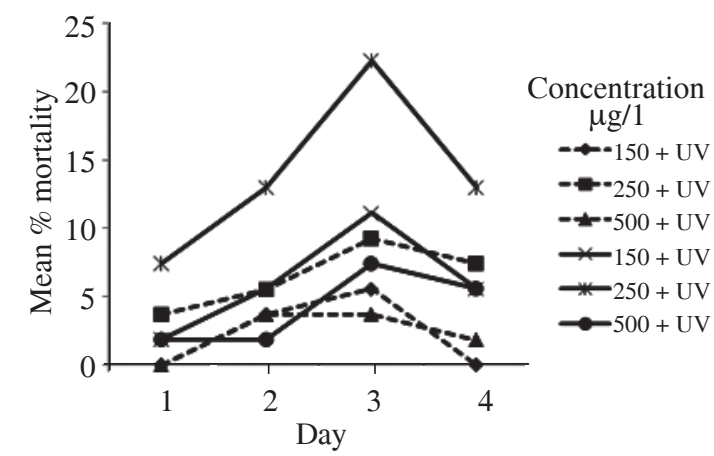

Figure 2: Variation in mortality rates (Mean \pm S.E.) (\% of tadpoles dead per day) in D. melanostictus larvae exposed to solar irradiated and non-irradiated carbofuran over a four day single exposure experiment.

As with mortality, irradiation reduced the potential of carbofuran to cause any growth impairment. By the end of the trial larvae exposed to photo-irradiated carbofuran were larger in size than those exposed to non-irradiated carbofuran (Table 1). A two sample t-test was used to examine if these differences in size between larvae exposed to irradiated and non -irradiated pesticides, were significant. The results confirmed that there was no significant difference in the initial lengths of larvae among control and treatment tanks ( $15 \pm-0.16, \mathrm{p}>0.05)$. However, the final body lengths of larvae at the end of four day experimental period in treatment tanks were significantly higher than those in the control tanks $(\mathrm{t} 9=-5.12, \mathrm{p}<0.05)$. 
Table 1: Mean body lengths ( \pm S.E) of D. melanostictus larvae exposed once to low, mid and high levels of non-irradiated and photo-irradiated carbofuran for four days.

\begin{tabular}{lcccc}
\hline Concentration & $\begin{array}{c}\text { Body lengths of control tadpoles } \\
\text { (non-irradiated carbofuran) } \\
(\mathrm{mm})\end{array}$ & $\begin{array}{c}\text { Body lengths of treated tadpoles } \\
\text { (photo irradiated carbofuran) } \\
(\mathrm{mm})\end{array}$ \\
\cline { 2 - 5 } & Initial & Final & Initial & Final \\
\hline $150 \mu \mathrm{g} 1^{-1}$ & 3.40 & 4.87 & 3.42 & 5.50 \\
& \pm 0.04 & \pm 0.13 & \pm 0.06 & \pm 0.06 \\
$250 \mu \mathrm{g} 1^{-1}$ & 3.42 & 4.78 & 3.41 & 5.39 \\
& \pm 3.41 & 5.26 & 3.41 & 5.58 \\
$500 \mu \mathrm{g} 1^{-1}$ & \pm 0.03 & \pm 0.27 & \pm 0.06 & \pm 0.04 \\
\hline
\end{tabular}

An interesting pattern was evident (Figure 3) such that the activity of larvae in control tanks (with non-irradiated pesticide) markedly increased at the beginning of the exposure and considerably declined by Day 4. In contrast, in the treatment tanks, although the larval activity did not increase initially, by Day 4 the activity of the tadpoles was higher than that of the controls. In both the control and treatment tanks, the variation in swimming activity across the three concentrations followed a similar pattern. The highest mean activity was shown by the larvae in $500 \mu \mathrm{gl}^{-1}$ of non-irradiated carbofuran, while the lowest was obtained for $250 \mathrm{\mu gl}^{-1}$ photo-irradiated carbofuran. Two sample t-tests conducted on the results of swimming activity Days 1 and 4 shows that there is a significant difference in larval activity patterns between control and treatment tanks (Day $1-\mathrm{t}_{11}=11.43, \mathrm{p}<0.05: \mathrm{t}_{10}=-2.49, \mathrm{p}<0.05$ ). Furthermore rough assessments of the number of tadpoles with abnormalities showed that while around $20 \%$ of the tadpoles exposed to non-irradiated carbofuran suffered from swollen heads and irregular swimming, only about $5 \%$ of tadpoles exposed to irradiated carbofuran showed such abnormalities.

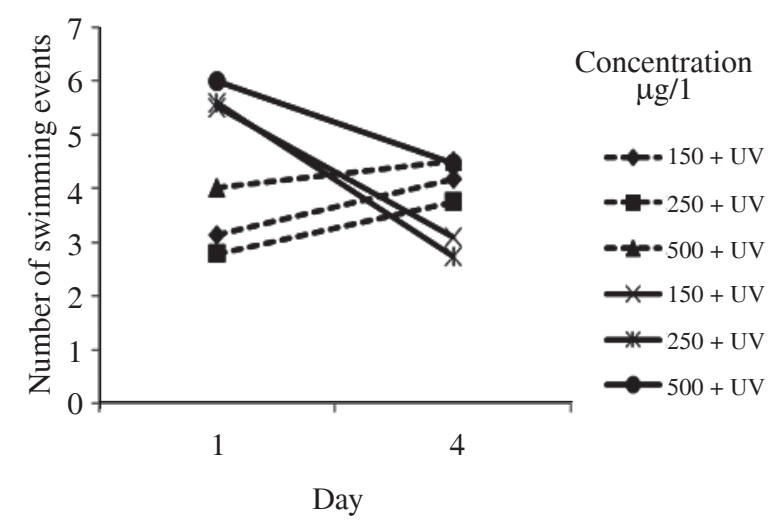

Figure 3: Swimming activity levels (per individual for 10 minutes) of D. melanostictus larvae exposed to different concentrations of non-irradiated and photo-irradiated carbofuran in the initial and final days of the experiment. 
Wijesinghe et al./Journal of Tropical Forestry and Environment Vol. 01, No. 01 (2011) 48-55

\section{Discussion}

The present study demonstrates that natural solar radiation has the potential to alter the toxicity of the carbamate pesticide carbofuran. This was evident from empirical trials that assessed the toxicity of carbofuran on tadpoles of the Asian Common Toad Duttaphrynus melanostictus. The reduction in toxic effects was noted with low, mid and high concentrations of carbofuran and with respect to three end points namely mortality, growth and activity of the tadpoles. For example, mortality observed in 250 $\mu \mathrm{gl}^{-1}$ of the photo-irradiated carbofuran (29\%) was lower than that which occurred when the pesticide was not irradiated $(55 \%)$. Trends in mortality were similar with both irradiated and non-irradiated carbofuran with mid concentrations resulting in higher levels of morality suggesting a hormetic response (Calabrese and Baldwin, 2002) commonly observed with endocrine disruptors, althoughthe exact mechanisms are poorly understood. Tadpoles exposed to irradiated carbofuran were also comparatively larger than those in control tanks. The activity patterns of the larvae over the experimental period also changed as a result of irradiation of the pesticide before exposure. Morphological deformities observed with non-irradiated carbofuran were more frequent than in those exposed to the irradiated pesticide.

As far as we are aware this is the first study that has demonstrated a reduction in toxicity of carbofuran by natural solar radiation. Considering other agrochemicals, Gupta et al., 2002 has shown reduced toxicity of the herbicide Atrazine on the Metolachlor bacterium Vibrio fischeri due to solar-irradiation. They have further demonstrated that in rivers and bays toxicity levels are negatively correlated with light intensity.

Carbofuran is generally degraded in water by chemical hydrolysis, microbial decomposition and photolysis (National Research Council of Canada, 1979) and has a half life of 27 days at $25^{\circ} \mathrm{C}$ and $\mathrm{pH} 7$ (DPR, 2002). Base-catalyzed hydrolysis results in the formation of the carbofuran-phenol and is recognized as the major degradation pathway of carbofuran in both water and sediment (Brahmaprakash et al., 1987; Seiber et al. 1978; Talebi and Walker, 1993; Yu et al. 1974). Carbofuran is also highly photodegradable (Raha and Das, 1990; Deuel et al., 1979); photomodification in water occurring as a result of ultraviolet light which produces high-energy photons (Larson, 1990). With regard to carbofuran, there is considerable potential for degradation from solar radiation because its absorbance is within the UV range (Iesce et al., 2005). It has been reported that photo-degraded products of carbofuran include 2,3-dihydro-2,2dimethylbenzofuran-4,7-diol and 2,3-dihydro-3 keto-2,2 dimethyl benzofuran-7-yl carbamate (or 3-ketocarbofuran) (Raha and Das, 1990). The toxicities of these by-products have not been previously evaluated. The lethal effects of carbofuran are mainly attributed to direct inhibition of acetylcholine esterase activity at central cholinergic synapses and neuromuscular junctions and the ultimate cause of death has been identified as respiratory failure (Hayes et al., 1991). The reduction in mortality observed in the present study suggests that that the by-products formed as a result of photodegradation may not be inhibiting acetylcholine esterase to the same degree as the carbofuran-phenol that is produced as a result of hydrolysis and hence is probably less toxic to the tadpoles.

In sharp contrast to the observations made in the present study, trials with another carbamate carbaryl on X. laevis and H. versicolor tadpoles (Zaga et al., 1998) and Rana sphenocephala (Boone and Bridges, 2003) have shown that solar radiation enhances toxic effects. Sarmah et.al., (2004) has reported that photodegradation of pesticides is influenced by the intensity and duration of the exposure to sunlight. The radiation levels used in the study with carbaryl $\left(4 \mathrm{i} \mathrm{W} / \mathrm{cm}^{2}\right)$ were, however, much lower than the 
ambient levels recorded for Sri Lanka $\left(189 \mathrm{~W} / \mathrm{m}^{2}\right)$, which was used in the present study. Thus it is very likely that further degradation of the toxic intermediate by-products into milder compounds occurs under high UV intensities. Additional causes of contradictory results may reflect differences in species tolerance, nature of exposure to the chemical (e.g. duration and frequency) and variations in pharmacokinetics of the pesticide under consideration.

In nature the effect of solar radiation may also be altered by other factors. For instance, it has been reported that the amount of dissolved organic matter in water tends to slower the rates of photolysis of pesticides making them more persistent in the environment (E.g. Bachman and Patterson 1999). Because of the complexity in natural ecosystems, where a combination of factors is often responsible for the observed effects in organisms, scientists reiterate that laboratory investigations may not directly simulate field conditions. While recognizing the importance of empirical trials in assessing toxicity, the present study signals a note of caution when directly extrapolating results of such trials to field scenarios. This study also emphasizes the necessity to investigate toxic effects under variable environmental conditions as this would substantially increase the applicability of results generated from laboratory trials to field situations.

\section{Acknowledgement}

We wish to acknowledge the University of Colombo, Sri Lanka, for financial assistance and laboratory facilities. We are also grateful to Mr. J. A. R. C. Jayakody and Ms. Sriyani Abeyratne for their assistance.

\section{References}

Bachman, J. and Patterson, H.H. (1999) Photodecomposition of the carbamate pesticide carbofuran : Kinetics and the influence of dissolved organic matter. Environmental Science \& Technology 33: 874881

Balasaraswathy, P. (2004). Sunlight in India, Skin Care N Cure. National Conference of Indian Association of Dermatology, Venereology and Leprosy, Mumbai. Available online at http:// www.skincarencure.com/sunlight.htm [ Accessed on 10th March 2009]

Blaustein, A. R. and Belden, L. K. (2003) Amphibian defences against ultraviolet-B radiation. Evolution and Development 5:89-97.

Boone, M. D. and Bridges, C. M. (2003) The interactive effects of UV-B and insecticide exposure on tadpole survival, growth and development. Biological Conservation 113:49-54.

Brahmaprakash, G.P., Panda, S. Sethunathan, N. (1987) Relative Persistance of Hexachlorocyclohexane, Methyl Parathion, and Carbofuran in an Alluvial Soil Under Flooded and Non-flooded conditions. Agricultural Ecosystems and the Environment 19:29-39.

Bretaud, C., Saglio, P., Saligaut, C., Auperin, B. (2002) Biochemical and behavioral effects of carbofuran in gold fish (Carassius auratus). Environmental Toxicology and Chemistry. 21, 175-181.

Calabrese, E.J. and Baldwin, L.A., (2002) Defining hormesis. Human and Experimental Toxicology 21: 91-98.

Campanella, L., Vitaliano, R. and Chimica, A. (2006) Toxicity Reduction Following H2O2/TiO2Photocatalyzed Reaction and Comparison with H2O2-Photolytic Reaction. ANNALI DI CHIMICA ROMA 97:123 - 134 . 
Deuel, L.E., Price, J.D., Turner, F.T. and Brown, K.W. (1979) Persistence of Carbofuran and its Metabolites, 3-Keto and 3-Hydroxy Carbofuran, under Flooded Rice Culture. Journal of Environmental Quality 8(1):23-26.

DPR (2002) Pesticide Chemistry Database. Environmental Monitoring Branch. Department of Pesticide Regulation, California. Available online at http://www.cdpr.ca.gov/docs/pur/purmain.htm [Accessed 5th march 2009]

Gosner K. L. (1960) A simplified table for staging anuran embryos and larvae with notes on identification. Herpetological Science 16: 183-190.

Gupta, G. Lin, Y. J., Karuppiah, M., Shaw, A. (2002) Effect of Simulated Sunlight on Atrazine and Metolachlor Toxicity of Surface Waters. Ecotoxicology and Environmental Safety 43:(1) 35-37.

Hayes, W. J., Laws, E. R., Baron, R. L. (1991) Carbamate insecticides: Handbook of Pesticide Toxicology, Academic Press, New York. P.3-6.

Iesce, M.R., Della Greca, M., Cermola, F., Rubino, M., Isidori, M. and Pascarella, L. (2005) Transformation and ecotoxicity of carbamic pesticides in water. Environmental Science and Pollution Research 13(2):105-109.

Kim, J., Cho, I., Zoh, K., Choi, K., (2007) Assessing the microbial toxicity of pentachlorophenolcontaminated water after treatment with UV radiation and $\mathrm{TiO} 2$ photocatalyst. Journal of Hazardous Materials 148: 281-286.

Kirtisinghe, P. (1955) The Amphibian of Ceylon .1st edition. Colombo, Charles circus.

Larson, R.A. (1990) Sensitized photo decomposition of organic compounds found in Illinois waste waters. University of Illinois, Institute for environmental studies, Urbana, Illinois.

National Research Council Canada. (1979) Carbofuran: Criteria for Interpreting the Effects of its Use on Environmental Quality. Publication \# NRCC 16740, Ottawa.

Oris, J. T. and Giesy, J. P. (1987) The photo induced toxicity of polycyclic aromatic hydrocarbons to larvae of the fathead minnow (Pimephales promelas). Chemosphere 16:1395-1404.

Raha, P. and Das, A.K. (1990) Photodegradation of carbofuran. Chemosphere 21:99-106.

Sarmah, A. K., Müller, K. and Ahmad, R. (2004) Fate and behaviour of pesticides in the agroecosystema review with a New Zealand perspective. Australian Journal of Soil Research 42(2):125-154.

Seiber, J.N., Catahan, M.P. and Barril, C.R. (1978) Loss of Carbofuran from Rice Paddy Water: Chemical and Physical Factors. Journal of Environmental Science and Health 13(2):131-148.

Sumanadasa, D.M.,M. R. Wijesinghe and W. D. Ratnasooriya 2008 Effects of diazinon on larvae of the Asian common toad Bufo melanostictus, Schneider 1799). Environmental Toxicolology and Chemistry. 27, 2320-2325.

Talebi, K. and Walker, C.H. (1993) A Comparative Study of Carbofuran Metabolism in Treated and Untreated Soils. Pesticide Science 39:65-69.

Tennakoon, S., Perera, B. and Haturusinghe, L. (2009) Intentional poisoning cases of animals with anticholinesterase pesticide-carbofuran in Sri Lanka. Legal Medicine 11:500-502.

WHO (2009) Ultraviolet radiation and the INTERSUN Programme. available online at http:// www.who.int/uv/intersunprogramme/activities/uv_index/en/index3.html [Accessed 2nd March 2009].

Yu, C.C., Booth, Gary, M., Hansen, Dale J. and Larsen, J.R. (1974) Fate of Carbofuran in a Model Ecosystem. Journal of Agriculture and Food Chemistry 22(3):431-434.

Zaga, A., Little, E.E., Rabeni, C.F. and Ellersieck, M.R. (1998) Photoenhanced toxicity of a carbamate insecticide to early life stage anuran amphibians. Environmental Toxicology and Chemistry 17(12):2543-2553. 